\title{
Solid para-hydrogen as the paradigmatic quantum crystal: Three observables probed by ultrahigh-resolution neutron spectroscopy
}

\author{
Felix Fernandez-Alonso, ${ }^{1, *}$ Carlos Cabrillo, ${ }^{2, \dagger}$ Ricardo Fernández-Perea, ${ }^{2}$ Francisco J. Bermejo, ${ }^{3}$ \\ Miguel A. González, ${ }^{4}$ Claudia Mondelli, ${ }^{5}$ and Emmanuel Farhi ${ }^{4}$ \\ ${ }^{1}$ ISIS Facility, Rutherford Appleton Laboratory, Chilton, Didcot, Oxfordshire OX11 OQX, United Kingdom \\ ${ }^{2}$ Instituto de Estructura de la Materia, IEM-CSIC, Serrano 123, Madrid E-28006, Spain \\ ${ }^{3}$ IEM-CSIC, Unidad Asociada de Física Aplicable, Department of Electricity and Electronics, \\ University of the Basque Country, P.O. Box 664, E-48080 Bilbao, Spain \\ ${ }^{4}$ Institut Laue Langevin, 6 rue Jules Horowitz F-38042, Grenoble Cedex 9, France \\ ${ }^{5}$ CNR-IOM-OGG Institut Laue Langevin, 6 rue Jules Horowitz F-38042, Grenoble Cedex 9, France
}

(Received 30 June 2012; revised manuscript received 5 September 2012; published 22 October 2012)

\begin{abstract}
Quantum crystals are characterized by zero-point-energy motions large enough to explore the details of the interaction potential beyond the harmonic approximation even at zero temperature. By making recourse to ultrahighresolution neutron spectroscopy, we have probed simultaneously three observables sensitive to anharmonic effects in solid para- $\mathrm{H}_{2}$ over a wide temperature range, namely, Bragg scattering, molecular root-mean-square displacements, and the spectral linewidth of the $J=0 \rightarrow 1$ rotational transition which, on average, amounts to a half-width at half-maximum of $1.8 \pm 0.2 \mu \mathrm{eV}$. These three quantities show no measurable variation with temperature, thus signaling the existence of a fully expanded crystal across the entire solid phase. Our results provide unambiguous experimental evidence for the intrinsic quantum character of this fundamental molecular solid.
\end{abstract}

DOI: 10.1103/PhysRevB.86.144524

PACS number(s): 67.80.ff, 61.05.F-, 67.10.Hk

\section{INTRODUCTION}

Quantum solids are characterized by large quantum fluctuations even at the lowest achievable temperatures, leading to a breakdown of the harmonic approximation. A particularly interesting example is the para- $\mathrm{H}_{2}$ cryocrystal since, by virtue of its molecular character and low mass, it represents a significant departure from the prototypical atomic systems ${ }^{4} \mathrm{He}$ and ${ }^{3} \mathrm{He}$. Owing to the low molecular weight of the $\mathrm{H}_{2}$ molecule, both intramolecular rotations and intermolecular translational degrees of freedom require a full quantum treatment from the outset to account for the properties of the liquid and solid phases at cryogenic temperatures. Moreover, the isotropy of the $J=0$ ground rotational state leads to a quantum-mechanical average that tends to minimize anisotropic effects. From these considerations, it follows that intermolecular interactions may be adequately described by an effective isotropic potential. ${ }^{1}$

The fundamental translational state of the para- $\mathrm{H}_{2}$ crystal corresponds to a hexagonal-closed-packed (hcp) structure known as Phase I, with a nearest-neighbour distance of $R_{N N} \sim$ $3.78 \AA$ (Refs. 1 and 2). In this phase, root-mean-square (rms) displacements associated with zero-point-energy motions of the molecular center of mass are large enough to explore the repulsive core of the interaction potential. As a consequence, the crystal is forced to expand to minimize its free energy. The above value for $R_{N N}$ is to be compared with $R \sim$ $3.41 \AA$, corresponding to the minimum of the $\mathrm{H}_{2}-\mathrm{H}_{2}$ two-body potential. ${ }^{1}$ Given such a remarkable $(\sim 30 \%)$ quantum-induced volume expansion, any attempt to calculate the spectrum of lattice frequencies under the Born-Karman approximation leads to unstable normal modes characterized by imaginary frequencies. These difficulties can be partly avoided by making recourse to self-consistent approaches, whereby an appropriate renormalization of two-body interactions may account for these quantum-mechanical effects. ${ }^{1}$ It is also well established that Phase I is characterized by quasifree molecular rotations where the total angular momentum $J$ associated with individual $\mathrm{H}_{2}$ molecules remains a good quantum number, yet little else is known about the underlying spectral line shapes.

Pioneering theoretical studies by Eliott and Hartmann ${ }^{3}$ and Van Kranendonk and coworkers ${ }^{4}$ have provided quantitative predictions for the spectral broadening of the first $J=0 \rightarrow$ 1 rotational transition in solid para $-\mathrm{H}_{2}$. These and subsequent works have identified the main broadening mechanism as arising from subtle crystal-field splittings dependent upon the phonon-field anisotropy. ${ }^{2}$ These quantities are both difficult to calculate and very sensitive to the delicate balance of forces experienced by a single molecule placed in a high-symmetry periodic lattice. On the experimental front, direct access to the linewidth of the (optically forbidden) $J=0 \rightarrow 1$ transition is an equally challenging task since the estimated linewidths are of the order of $\mu \mathrm{eV}$ at a spectral transition energy of $\sim 14.6 \mathrm{meV}$.

In this work, we use state-of-the-art inelastic neutron scattering techniques to provide direct measurement of the $J=$ $0 \rightarrow 1$ linewidth as a function of temperature in solid para $-\mathrm{H}_{2}$. This spectroscopic study is complemented by measurements of the lattice structure and molecular form factors. These data allow for a direct and quantitative assessment of the quantum nature of the para- $\mathrm{H}_{2}$ crystal, thus enabling a systematic study of anharmonic effects in this fundamental molecular solid.

\section{EXPERIMENT}

All measurements were performed on the IN20 thermal neutron spectrometer at the Institut Laue Langevin, France. ${ }^{5}$ The instrument was used either in polarized triple-axis (TAS) mode or in TAS-spin-echo (TASSE) mode. ${ }^{6,7}$ In TAS mode, 
two spin flippers located before (flipper F1) and after (F2) the sample were used to implement polarization analysis. In both the TAS and TASSE experiments, a final wave vector of $k_{f}=2.66 \AA^{-1}$ was chosen to maximize the available incident flux at energy transfers $\sim 15 \mathrm{meV}$ in neutron energy loss. In TASSE mode, the accessible Fourier time spans $3.2 \mathrm{~ns}$ at $k \sim 2.7 \AA^{-1}$.

The sample cell consisted of two concentric Al cylinders with a height and external diameter of 50 and $25 \mathrm{~mm}$, respectively. Gas was injected into this sample cell using transfer lines with an internal diameter of $2 \mathrm{~mm}$. The sample cell was housed inside a pumped helium cryostat to achieve base temperatures around $2 \mathrm{~K}$. During the measurements, a neutron beam of area $20 \times 20 \mathrm{~mm}^{2}$ illuminated the central part of this sample holder.

Molecular para $-\mathrm{H}_{2}$ was prepared offline from normal$\mathrm{H}_{2}$ (Linde, 99.999\% purity) using a second cryostat. A cylindrical gas cell of $15-\mathrm{mm}$ inner diameter and $65-\mathrm{mm}$ height was connected to the gas-transfer system described above. Following the procedures described by Sullivan and coworkers, ${ }^{8}$ the gas cell was half filled with 6 grams of Oxisorb, a commercial preparation which consists of silica gel impregnated with the paramagnetic salt $\mathrm{CrO}_{3}$. This chromiumoxide catalyst acts as an excellent ortho-to-para hydrogen converter. ${ }^{8}$ Thermal heaters and sensors were placed at the top and bottom of the cell and normal $-\mathrm{H}_{2}$ was condensed in the presence of the Oxisorb gel. The temperature at the top of the cell was stabilized to $15 \mathrm{~K}$ while the bottom one was set to $19 \mathrm{~K}$. The corresponding thermal gradient across the cell induced convective currents inside the cylinder aimed at forcing the uniform and complete mixing of the liquid and catalyst. The resulting liquid $\mathrm{H}_{2}$ was left under these conditions over a period of five days. Neat para- $\mathrm{H}_{2}$ was then transferred to a one-liter reservoir by crossing the boiling point curve below the critical temperature of $\mathrm{H}_{2}(\sim 33 \mathrm{~K})$. Immediately after this step, para- $\mathrm{H}_{2}$ was gently transferred from the reservoir to the sample cell while slowly cooling the latter to $13 \mathrm{~K}$. Consistent with our own experience as well as with previous works on high-purity parahydrogen, ${ }^{9-11}$ this simple procedure avoided the formation of polycrystalline specimens.

All neutron measurements on solid para- $\mathrm{H}_{2}$ up to the triple point $\left(T_{t p}=13.8 \mathrm{~K}\right)$ were performed immediately after the crystallization procedure described above. The sample pressure was also monitored and remained always below its value at the triple point $\left(P_{t p}=72 \mathrm{mbar}\right)$. Following these measurements in the solid, additional measurements were conducted in the liquid phase.

\section{RESULTS}

At a sample temperature $T=13.2 \mathrm{~K}$, we performed rough elastic- $Q$ scans (momentum transfer step $\Delta Q=0.1 \AA^{-1}$ ) in the TAS mode with F1 in the OFF state. In this configuration, $\mathrm{F} 2$ in the ON state is only sensitive to incoherent scattering, while in the OFF state both coherent and incoherent scattering contribute to the observed signal. ${ }^{12}$ Molecules in the para ground state only scatter coherently (weakly) below the $0 \rightarrow 1$ transition, whereas ortho- $\mathrm{H}_{2}(J=1)$ also scatters incoherently (strongly). As shown in Fig. 1, the F2-OFF data display two

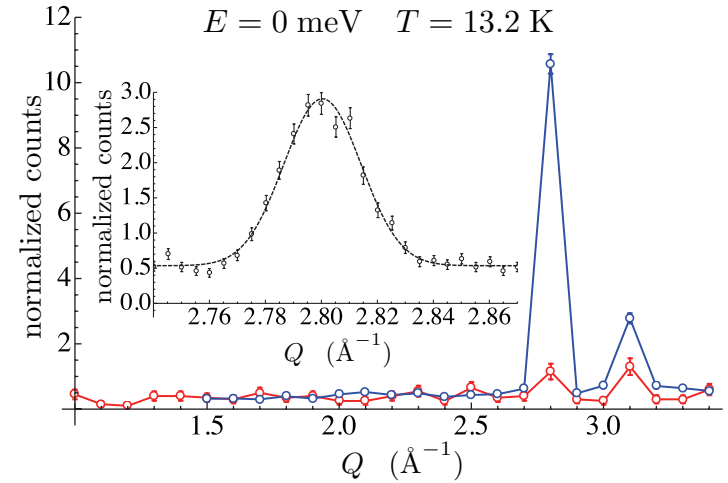

FIG. 1. (Color online) Elastic- $Q$ scans in TAS mode (F1-OFF) on the para $-\mathrm{H}_{2}$ crystal at $T=13.2 \mathrm{~K}$. The blue/red data correspond to F2-OFF/ON scans with a $Q$ step of $0.1 \AA^{-1}$. The inset shows a finer scan with a $Q$ step of $0.005 \AA^{-1}$. For further details see the text.

peaks at high $Q$. The one located at $\sim 3.1 \AA^{-1}$ arises from the $\mathrm{Al}$ container. The other, located at $\sim 2.8 \AA^{-1}$, comes from the para $-\mathrm{H}_{2}$ crystal. This feature corresponds to the (102) reflection of the hcp structure of para- $\mathrm{H}_{2}$ (Ref. 13). The systematic absence of three additional Bragg reflections over this momentum-transfer range signals the presence of a highquality single-crystal specimen characterized by macroscopic coherence lengths.

A comparison between the F2-OFF and F2-ON scans also confirms the $J=0$ character of the crystal since both F2$\mathrm{ON} / \mathrm{OFF}$ signals are indistinguishable below the Bragg peak. A fit of the expected difference between the F2-ON/OFF datasets gives an ortho concentration of $c_{o}=0.001 \pm 0.002$, indicating a high-purity para- $\mathrm{H}_{2}$ specimen.

Aiming at a precise determination of the peak position, we performed a finer F2-OFF scan centered at $Q=2.8 \AA^{-1}$. The result is shown in the inset of Fig. 1. A Gaussian fit yields a peak position $Q_{p}=2.8004 \pm 0.0008 \AA^{-1}$ where the error bars denote a $2 \sigma(95 \%)$ confidence band. This fine-grained $Q$ scan was repeated at a temperature $T=3.1 \mathrm{~K}$ giving a peak position $Q_{p}=2.8023 \pm 0.0006 \AA^{-1}$. From these diffraction data, we obtain an expansion ratio of $Q_{p}(13.17) / Q_{p}(3.1)=1.0007$. In terms of the triple-point temperature $T_{t p}=13.8 \mathrm{~K}$ (Ref. 14), the temperatures of our diffraction data correspond to 0.22 and $0.95 T_{t p}$, respectively, range over which thermal lattice expansion is negligibly small.

At this point, it is worth comparing these results with those from a classical simulation using the isotropic SilveraGoldman potential. ${ }^{15}$ Our simulations show that such a computational crystal melts at a much higher temperature of $\sim 25 \mathrm{~K}$, as expected from classical law-of-corresponding-states arguments. Nonetheless, and as Lindemann pointed out long ago, ${ }^{16}$ molecular displacements determine the stability of a crystal. Indeed, our computational crystal does melt when it reaches a rms molecular displacement $\sim 0.75 \AA$. As shown in more detail below, this rms value coincides with the one measured in this work. The equivalent thermal-expansion ratio of this classical crystal [i.e., $Q_{p}\left(0.95 T_{t p}\right) / Q_{p}\left(0.22 T_{t p}\right)$ ] amounts to 1.04 , to be compared with our experimental value of 1.0006. This thermal expansion ratio is also smaller than the value reported by Kruspskii and coworkers using x-ray diffraction $\left[Q_{p}(13.17) / Q_{p}(3.1)=1.003\right]$ (Ref. 17). Such a 
discrepancy may be ascribed to significant differences in sample-preparation protocols compared to the present work. Kruspskii and coworkers studied a polycrystalline specimen grown within the pores of a snowy solid-krypton matrix. Ortho contamination was estimated to be of the order of $\sim 0.2 \%$, yet it was not measured in situ. Moreover, polycrystallinity ensured access to the first three Bragg peaks of the $\mathrm{H}_{2}$ hcp lattice, while the fcc (111) reflection of the solid-krypton matrix provided an internal and absolute calibration of their momentum-transfer scale. Although this procedure is a very convenient one for precise structural studies, it may also lead to an increase in sensitivity to thermal effects, as explained in more detail in the Discussion.

The spectral response around the first rotational excitation at energy transfers $\sim 14.6 \mathrm{meV}$ was studied in detail by performing inelastic TAS scans with F2-ON at a fixed momentum transfer $Q=2.5 \AA^{-1}$. In this case, only the scattering from molecules that are rotationally excited by the incident neutron beam can contribute to the signal (purely incoherent-scattering events). Under these conditions, the measured intensities are proportional to an incoherent scattering law of the form

$$
S_{\text {inc }}(Q, E)=e^{-\frac{\left\langle u^{2}\right\rangle}{3} Q^{2}} \delta\left(E-E_{01}\right)+S_{\text {vib }}\left(Q, E-E_{01}\right),
$$

where $u$ is the molecular displacement and $S_{\mathrm{vib}}(Q, E)$ is the vibrational contribution to the dynamic structure factor. We have assumed isotropy in $u$ and no coupling between the rotational and translational modes. On the basis of this model, we find a well-resolved resolution-limited Gaussian contribution at a rotational energy $E_{01}=14.64 \pm 0.03 \mathrm{meV}$ with a full width at half maximum (FWHM) of $1.3 \mathrm{meV}$. These figures provide an estimate of the requisite precision of our experimental setup to define a net energy transfer centered at $\sim 14.6 \mathrm{meV}$ in subsequent TASSE measurements.

A series of $Q$ scans at a fixed energy transfer of $E=$ $14.6 \mathrm{meV}$ allowed a systematic study of the temperature dependence of molecular rms displacements. In this case, the observed signal is proportional to the energy integral below the resolution-broadened rotational peak. Taking into account the quantum-mechanical form factor of the para- $\mathrm{H}_{2}$ molecule, ${ }^{18}$ the scattered intensities are proportional to

$$
I(Q)=j_{1}(Q d / 2) \exp \left(-\frac{\left\langle u^{2}\right\rangle}{3} Q^{2}\right),
$$

where $j_{1}(x)$ is the first-order spherical Bessel function and $d$ is the internuclear distance of $\mathrm{H}_{2}$. The inset in Fig. 2 shows a representative inelastic $Q$ scan and the corresponding fit using Eq. (2). We have explored a total of seven temperatures above and below the melting temperature of the crystal. The behavior of the rms displacement with temperature is also displayed in the figure. As expected, on crossing the triple-point temperature, there is a sudden jump signaling the melting of the crystalline phase. Below melting, molecular rms displacements remain essentially constant at $\sim 0.75 \AA$. Rosciszewski and Paulus ${ }^{19}$ have recently calculated self-consistently rms displacements as a function of nearest-neighbour distance $R_{N N}$. For an hep structure with $R_{N N}=3.78 \AA$, they obtained $\sqrt{\left\langle u^{2}\right\rangle}=0.73 \AA$, in excellent agreement with the present study.

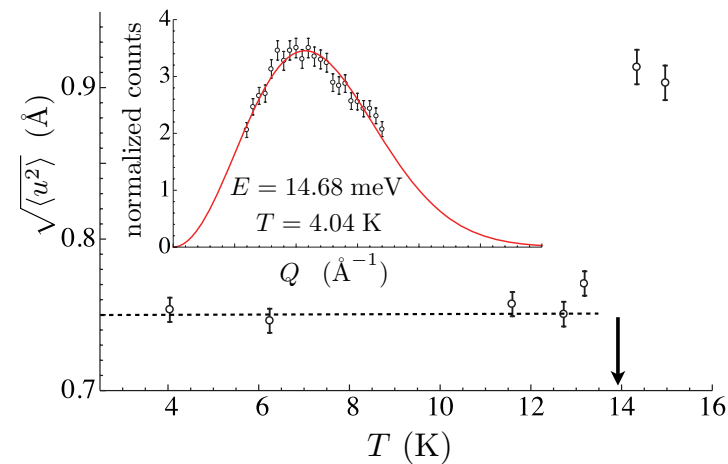

FIG. 2. (Color online) Temperature dependence of $\sqrt{\left\langle u^{2}\right\rangle}$. The arrow marks the triple-point temperature $\left(T_{t p}=13.8 \mathrm{~K}\right)$. The dashed line corresponds to $\sqrt{\left\langle u^{2}\right\rangle}=0.75 \AA$. The inset shows a $Q$ scan at $E=14.68 \mathrm{meV}$ and $T=4.04 \mathrm{~K}$. The red solid line corresponds to a fit using Eq. (2) with $d=0.74 \AA$.

Our value of $\sim 0.75 \AA$ for the molecular rms displacement also compares favorably with $\sqrt{\left\langle u^{2}\right\rangle}=0.69 \AA$ A reported by Nielsen at $T=5.4 \mathrm{~K}$ some time ago. ${ }^{20}$

Keeping the same TAS configuration as above, TASSE measurements were performed over the temperature range $T=3-15 \mathrm{~K}$. These results are reported in Fig. 3. The TASSE amplitudes shown in Fig. 3 are adequately described by a single-exponential decay of the form $e^{-\Gamma t}$ (see the inset in Fig. 3). In these time-resolved measurements, instrumental resolution effects result in an increase of the spin-echo amplitude decay by $e^{-\Gamma_{\text {res }} t}$ and, therefore, the intrinsic lifetime $\tau$ of the inelastic $J=0 \rightarrow 1$ transition is given by $\tau^{-1}=\Gamma-\Gamma_{\text {res }}$. These spin-echo decay measurements are very time consuming, requiring over one day per temperature. Following these full-decay measurements (filled symbols), we cooled the sample back to base and performed quicker four-point TASSE scans over the same temperature range prior to crossing the triple point into the liquid phase. The open symbols in Fig. 3 show the results of this second sweep

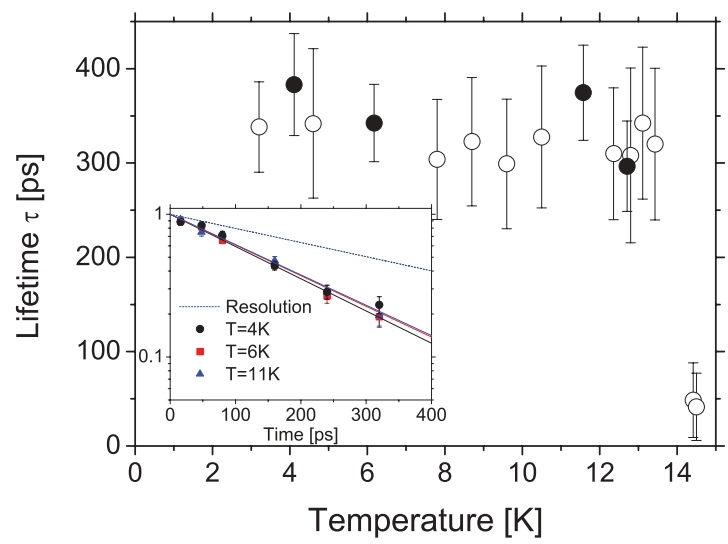

FIG. 3. (Color online) Lifetime of the $J=0 \rightarrow 1$ transition as a function of temperature. The inset shows TASSE amplitudes as a function of spin-echo time (closed symbols) and their exponential fits (solid lines) at several temperatures. The blue (dashed) line corresponds to the instrumental resolution obtained with a silicon standard. 
referenced to our previous TASSE data at $T=6.2 \mathrm{~K}$. Within the uncertainty of the measurements, the spectral linewidth of the $J=0 \rightarrow 1$ transition remains constant with temperature below melting with an average value of $\hbar / \tau=1.8 \pm 0.2 \mu \mathrm{eV}$. In agreement with what has been observed with the Bragg reflections and molecular rms displacements, only the melting of the crystal results in a measurable change in spectral linewidths.

\section{DISCUSSION}

It is instructive at this juncture to compare the present TASSE results with previous attempts to obtain this quantity using other spectroscopic probes. Estimates of the energy splitting $V_{M}$ between $M=0$ and $M= \pm 1$ Zeeman sublevels of isolated ortho- $\mathrm{H}_{2}(J=1)$ in a solid para $-\mathrm{H}_{2}$ matrix have been obtained in nuclear-magnetic-resonance (NMR) experiments at very low temperatures $(T<1 \mathrm{~K})$. These measurements are spread over a wide range $\left(V_{M}=0.4-4.0 \mu \mathrm{eV}\right)$, yet their magnitude agrees well with our average TASSE value of $1.8 \pm 0.2 \mu \mathrm{eV}$. The value of $V_{M}=0.7 \pm 0.2 \mu \mathrm{eV}$ reported by Meyer and coworkers ${ }^{21,22}$ some time ago is still regarded as the most reliable one using NMR techniques. Using laser spectroscopy on ortho- $\mathrm{H}_{2}$-doped solid para- $\mathrm{H}_{2}$, Dickson and coworkers ${ }^{23,24}$ derived a crystal-field splitting $V_{M}=1.0 \mu \mathrm{eV}$ from an analysis of overtone transitions from the ground vibrational levels. These authors have argued that such a splitting is consistent with the anisotropy inherent to dispersion and induction interactions without the need to invoke the renormalization of phonon modes. In either case, experimental access to $V_{M}$ prior to the present neutronscattering results has required the use of small amounts of ortho $-\mathrm{H}_{2}$ in an otherwise para- $\mathrm{H}_{2}$ lattice, as neither para- $\mathrm{H}_{2}$ in its ground rotational state is NMR active nor its associated $J=0 \rightarrow 1$ rotational transition is optically allowed. From this comparison, we conclude that the doping protocols required to perform NMR or optical experiments have a measurable (yet relatively small) effect on the resulting linewidth of this spectral transition. The present TASSE measurements also represent a two-order-of-magnitude improvement over the highest spectral resolution reported to date in neutron studies on bulk and adsorbed $\mathrm{H}_{2}$ using the IRIS spectrometer at the ISIS Facility, United Kingdom. ${ }^{25-27}$ These previous works were able to attain spectral widths of $\sim 100 \mu \mathrm{eV}$ at an energy transfer of $15 \mathrm{meV}$, and are representative of the current limits of resolution using non-spin-echo neutron spectroscopy.

The picture that emerges from our experimental results is that of a crystal that is essentially immune to thermal effects. The origin of such a unique physical behavior can be rationalized by first considering the liquid state above melting. Because of the strong metastability of the fundamental para state at cryogenic temperatures, liquid $\mathrm{para}-\mathrm{H}_{2}$ near the triple point behaves as a simple liquid characterized by isotropic interaction potentials (i.e., essentially free from the action of intramolecular degrees of freedom). Well before crystallization, quantum effects associated with translational degrees of freedom are already strong, as evidenced by a marked depression of the triple-point temperature $\left(T_{t p}=13.8 \mathrm{~K}\right)$ relative to classical predictions $(25 \mathrm{~K})$ as a result of additional contributions to the kinetic energy arising from the Heisenberg uncertainty principle. This behavior has been verified in previous experimental ${ }^{28,29}$ studies and results in a remarkable isothermal dependence of the kinetic energy on density. ${ }^{30}$ As in the case of the crystal, this phenomenology can be understood in terms of the single-molecule wave function exploring the repulsive core of the intermolecular potential. Similarly, owing to the effects of particle delocalization on the overall magnitude of attractive intermolecular interactions, liquid para $-\mathrm{H}_{2}$ near its triple point can sustain collective excitations which are otherwise absent in the classical computational (supercooled) counterpart. ${ }^{31}$ All things considered, it might be argued that liquid para- $\mathrm{H}_{2}$ represents the quintessential quantum Boltzmann liquid where wave-like dynamics clearly dominate over thermal effects near the triple point. Notwithstanding the above, crystallization is still possible under such circumstances. By adopting a close-packed structure, the system maximizes nearest-neighbor distances, minimizes repulsive interactions, and, in so doing, the attractive part of the potential can lead to a lower free energy relative to that of the liquid. When such a possibility is advantageous, the crystal will remain fully expanded irrespective of temperature, as intermolecular repulsive forces are driven by quantum delocalization from the outset. Paradoxically, the prototypical manifestation of anharmonicity, that is, crystal expansion, is absent in spite of the intrinsic anharmonic dynamics of the system.

Some immediate consequences also follow from the above considerations. Since quantum coherence is such a fragile property, small deviations from ideal conditions could very well translate into the reemergence of the expected behavior for a solid, including thermal expansion. As in the case of the work by Krupskii and coworkers, ${ }^{17}$ these deviations may be caused by the presence of significant amounts of ortho nuclear-spin impurities in the lattice, or the use of a porous matrix leading to the disruption of crystal structure at the mesoscale and the presence of additional interactions with the matrix material. Likewise, even in the most pristine para $-\mathrm{H}_{2}$ sample, pressure is also expected to increase the possibility of thermal effects. Indeed, Kuroda and coworkers ${ }^{32}$ have observed the shift and broadening of Raman features associated with vibrational levels as a function of temperature at pressures of $\sim 30$ bar. More intriguing is the possibility of the effective quantum annealing of quenched defects and disorder. As opposed to quantum tunneling that would naturally lead to exponentially long "annealing" times, quantum fluctuations exploring the repulsive core of the interaction potential are present from the outset following crystallization. Such a preponderance of quantum fluctuations could, in principle, preclude the possibility of bulk vitrification upon cooling. Certainly, the well-known ease to produce high-quality para- $\mathrm{H}_{2}$ crystals over macroscopic coherence lengths ${ }^{9-11}$ is consistent with such a possibility.

In summary, we have reported simultaneous measurement of the natural linewidth of the lowest-lying rotational transition, root-mean-squared molecular displacements, and lattice structure in crystalline para- $\mathrm{H}_{2}$. Both molecular rms displacements and rotational linewidths are insensitive to temperature, with average values of $0.75 \AA$ and $1.8 \pm 0.2 \mu \mathrm{eV}$, 
respectively. Our results depict a crystal lattice subjected to full quantum dynamics.

\section{ACKNOWLEDGMENTS}

Work supported in part by Grant No. MAT2007-65711C04-01 from the Spanish Ministerio de Ciencia e Innovación.
We thank the Institut Laue-Langevin for the provision of beam time and technical assistance, particularly from $\mathrm{M}$. Enderle and the Cryogenics Team. FFA gratefully acknowledges the UK Science \& Technology Facilities Council for financial support and Evgeniy Goremychkin for the translation of Ref. 17 from the original Russian. *felix.fernandez-alonso@stfc.ac.uk; also at Department of Physics and Astronomy, University College London, Gower Street, London, WC1E 6BT, United Kingdom.

†ccabrilo@foton0.iem.csic.es

${ }^{1}$ I. F. Silvera, Rev. Mod. Phys. 52, 393 (1980).

${ }^{2}$ V. G. Manzhelii and Y. A. Freiman, Physics of Cryocrystals (American Institute of Physics, New York, 1997).

${ }^{3}$ R. J. Elliot and M. W. Hartmann, Proc. Phys. Soc. London 90, 671 (1967).

${ }^{4}$ J. Van Kranendonk, Solid Hydrogen: Theory of the Properties of Solid $\mathrm{H}_{2}, H D$, and $D_{2}$ (Plenum, New York, 1983).

${ }^{5} \mathrm{http}$ ///www.ill.eu/instruments-support/instruments-groups/ instruments/in20/.

${ }^{6}$ C. M. E. Zeyen, J. Phys. Chem. Solids 60, 1573 (1999).

${ }^{7}$ C. M. E. Zeyen and P. C. Rem, Meas. Sci. Technol. 7, 782 (1996).

${ }^{8}$ N. S. Sullivan, D. Zhou, and C. M. Edwards, Cryogenics 30, 734 (1990).

${ }^{9}$ R. G. Bohn and C. F. Mate, Phys. Rev. B 2, 2121 (1970).

${ }^{10}$ O. A. Korolyuk, B. Ya. Gorodilov, A. I. Krivchikov, A. S. Pirogov, and V. V. Dudkin, J. Low Temp. Phys. 111, 515 (1998).

${ }^{11}$ F. Köenigsmann, M. Fushitani, N. Owschimikow, D. T. Anderson, and N. Schwentner, Chem. Phys. Lett. 458, 303 (2008).

${ }^{12}$ G. L. Squires, Introduction to the Theory of Thermal Neutron Scattering (Dover, New York, 1996).

${ }^{13}$ C. S. Barret, L. Meyer, and J. Wassernman, J. Chem. Phys. 45, 834 (1966).

${ }^{14}$ N. Sakoda, K. Shindo, K. Shinzato, M. Kohno, Y. Takata, and M. Fujii, Int. J. Thermophys. 31, 275 (2010).

${ }^{15}$ I. F. Silvera and V. V. Goldman, J. Chem. Phys. 69, 4209 (1978).

${ }^{16}$ F. A. Lindemann, Phys. Z. 11, 609 (1910).
${ }^{17}$ I. N. Krupskii, A. I. Prokhvatilov, and G. N. Shcherbakov, Fiz. Nizk. Temp. 9, 83 (1983).

${ }^{18}$ V. F. Sears, Can. J. Phys. 127, 1279 (1966).

${ }^{19}$ K. Rosciszewski and B. Paulus, Mol. Phys. 108, 2147 (2010).

${ }^{20}$ M. Nielsen, Phys. Rev. B 108, 1626 (1973).

${ }^{21}$ S. Washburn, R. Schweizer, and H. Meyer, J. Low Temp. Phys. 40, 187 (1980).

${ }^{22}$ R. Schweizer, S. Washburn, and H. Meyer, Phys. Rev. Lett. 40, 1035 (1978); 41, 913 (1978).

${ }^{23}$ R. M. Dickson, T. Momose, T. J. Byers, and T. Oka, Phys. Rev. B 57, 941 (1998).

${ }^{24}$ R. M. Dickson, T. J. Byers, and T. Oka, J. Low Temp. Phys. 102, 241 (1996).

${ }^{25}$ F. Fernandez-Alonso, F. J. Bermejo, C. Cabrillo, R. O. Loutfy, and M. L. Saboungi, Phys. Rev. Lett. 98, 215503 (2007).

${ }^{26}$ A. Lovell, F. Fernandez-Alonso, N. T. Skipper, K. Refson, S. M. Bennington, and S. F. Parker, Phys. Rev. Lett. 101, 126101 (2008).

${ }^{27}$ F. Fernandez-Alonso, F. J. Bermejo, and M. L. Saboungi, in Handbook of Nanophysics, edited by K. D. Sattler, Vol. 5 (CRC, Boca Raton, FL, 2010).

${ }^{28}$ W. Langel, D. L. Price, R. O. Simmons, and P. E. Sokol, Phys. Rev. B 38, 11275 (1988).

${ }^{29}$ M. Celli, D. Colognesi, and M. Zoppi, Eur. Phys. J. B 14, 239 (2000).

${ }^{30}$ M. Zoppi, D. Colognesi, and M. Celli, Eur. Phys. J. B 23, 171 (2001).

${ }^{31}$ F. J. Bermejo, K. Kinugawa, C. Cabrillo, S. M. Bennington, B. Fak, M. T. Fernandez-Diaz, P. Verkerk, J. Dawidowski, and R. Fernandez-Perea, Phys. Rev. Lett. 84, 5359 (2000).

${ }^{32}$ K. Kuroda, A. Koreeda, S. Takayanagi, M. Suzuki, and K. Hakuta, Phys. Rev. B 67, 184303 (2003). 